\title{
A INCORPORAÇÃO DO BIM NO PROCESSO DE TRABALHO DO COORDENADOR DE PROJETOS
}

\author{
OLEGÁRIO, Wylliane Ladislau Coelho \\ Universidade Federal da Paraíba, e-mail: wyllianeladislau@hotmail.com \\ NÓBREGA JUNIOR, Claudino Lins \\ Universidade Federal da Paraíba, e-mail: claudinolins@hotmail.com
}

\begin{abstract}
RESUMO
O Building Information Modeling, BIM, representa um grande avanço na indústria da Arquitetura, Engenharia e Construção, e funciona com um conjunto de processos e tecnologias, resultando em uma nova metodologia para todo o processo do projeto. O coordenador de projetos já tem seu escopo de trabalho definido, mas, com a difusão do BIM no mercado de trabalho, sua função pode demandar novas atribuições, exigindo novas características e conhecimentos. Este trabalho, fruto de um Trabalho de Conclusão de Curso, finalizado, tem como objetivo avaliar como a inserção do BIM no mercado pode impactar nas atribuições do coordenador de projetos. Para tal, foram realizadas duas entrevistas com um coordenador de projetos de uma construtora na cidade de João Pessoa. Como resultado, foi possível observar que as atividades exigidas pelo BIM foram incorporadas a função do coordenador de projetos, e as maiores dificuldades para tal função são a falta de treinamento dos profissionais. Por fim, pode-se verificar a importância do BIM, e como essa ferramenta pode contribuir significativamente para a eficiência da construção civil.
\end{abstract}

Palavras-chave: BIM, Coordenador de Projetos, Construção.

\begin{abstract}
Building Information Modeling, BIM, represents a breakthrough in the Architecture, Engineering and Construction industry, and works with a set of processes and technologies, resulting in a new methodology for the entire design process. The project coordinator already has a defined scope of work, but with the dissemination of BIM in the labor market, its role may require new assignments, requiring new characteristics and knowledge. This work, the result of a Completed Course Completion Work, aims to evaluate how the insertion of BIM in the market can impact the assignments of the project coordinator. For that, two interviews were conducted with a project coordinator of a construction company in the city of João Pessoa. As a result, it was possible to observe that the activities required by BIM were incorporated into the role of the project coordinator, and the greatest difficulties for such a function are the lack of training of professionals. Finally, one can verify the importance of BIM, and how this tool can contribute significantly to the efficiency of civil construction.
\end{abstract}

Keywords: BIM, Project Coordinator, Construction.

\section{INTRODUÇÃO}

A Câmara Brasileira de Indústria e Construção (CBIC, 2016), define Building Information Modeling (BIM), como "um conjunto de políticas, processos e tecnologias que, combinados, resultam em uma metodologia para gerenciar o processo de projetar de uma edificação, testar seu desempenho, e

OLEGÁRIO, W. L. C.; NÓBREGA JUNIOR, C. L. A incorporação do BIM no processo de trabalho do coordenador de projetos. In: SIMPÓSIO BRASILEIRO DE QUALIDADE DO PROJETO NO AMBIENTE CONSTRUÍDO, 6., 2019, Uberlândia. Anais... Uberlândia: PPGAU/FAUeD/UFU, 2019. p 596-601. DOI https://doi.org/10.14393/sbqp19055. 
gerenciar as suas informações utilizando plataformas digitais". Manzione (2013) ainda complementa, caracterizando-o como "um processo que permite a gestão da informação".

O BIM é uma das novidades no que diz respeito a otimização e inovação. Estudos feitos por Wanderley (2017) retratam a situação da Indústria da Arquitetura, Engenharia e Construção, e mostram que a adesão dessas empresas ao BIM é muito pequena.

O coordenador de projetos, já tem sua função estabelecida e difundida no mercado, gerenciando toda a equipe no processo do projeto. Conforme Nóbrega Júnior (2012), a coordenação de projetos é uma atividade de grande importância, e como tal, demanda complexidade, exigindo de seus indivíduos habilidades e atributos específicos para sua eficácia no processo de trabalho. Olegário (2018), destaca que ainda há muitos questionamentos ao integrar as funções do coordenador de projetos com o BIM.

Nesse contexto, este trabalho tem como objetivo analisar a inserção do BIM no escopo de trabalho do coordenador de projetos, e mostrar a importância desta ferramenta para o aumento da produtividade na indústria.

\section{REFERENCIAL TEÓRICO}

A coordenação de projetos, segundo Melhado (2001) tem como objetivo não só os serviços de projeto, mas também o controle da execução, compreendendo responsabilidades em todas as fases do empreendimento.

Já de acordo como Fabrício (2002), coordenação de projetos dá suporte ao desenvolvimento dos projetos, visando garantir sua compatibilidade, bem como os requisitos estabelecidos para o empreendimento inicial.

Já para Olegário (2018), a coordenação de projetos pode ser entendida então como a gestão sistemática de todos os profissionais envolvidos na elaboração de projetos de um empreendimento.

Portanto, a coordenação de projetos se insere de maneira a potencializar o desenvolvimento dos projetos, trabalhando em sincronia com os projetistas de edificações, ao mesmo tempo em que possibilita a visualização macro da construção, pensando a curto, médio e longo prazo, proporcionando uma maior integração entre todas as fases do empreendimento.

Nóbrega Júnior (2012) destaca que o coordenador deve possuir habilidades e atributos específicos para sua eficácia, e conforme Olegário (2018) pontua, com a inserção com BIM no mercado, as características mudam, o ritmo de trabalho é outro, as facilidades proporcionadas por essa ferramenta são inúmeras.

O BIM, surge como uma ferramenta, segundo Olegário (2018), uma ferramenta que integra todos os tipos de projetos, sendo ainda necessária a figura do coordenador, que direciona e relaciona o trabalho de todos os projetistas envolvidos.

Manzione (2013) destaca que com o BIM há uma maior facilidade de comunicação entre os projetistas, bem como a compatibilização dos projetos se torna mais simples e ágil. Entretanto, o mesmo ainda pontua a necessidade da gestão eficaz entre o processo do projeto e sua modelagem para o bom 
funcionamento de tudo que o BIM proporciona, o que pode ser traduzido na necessidade do coordenador de projetos para essa gestão.

Ao passo que o BIM se insere e evolui no mercado, um coordenador está cada vez mais requisitado, porém, com suas funções voltadas as necessidades que o contexto BIM requer. Todavia, a implantação e gestão do BIM vêm demandando habilidades específicas dos profissionais, e, por isso, têm surgido no mercado novos especialistas, Barison e Santos (2010) destacam que a principal função desse profissional é gerenciar as pessoas na implementação e/ou na manutenção do processo em BIM. Manzione (2013) estabelece esse profissional como BIM Manager, ou gerente de BIM.

Desse modo, o BIM Manager deve ter visão disciplinar, e conforme Barison e Santos (2010), deve assumir as responsabilidades sobre treinamentos, softwares e as demais necessidades da implantação BIM.

Com isso, há duas possibilidades na relação do coordenador de projetos com o BIM, a cooperação dos dois profissionais: o coordenador de projetos e o BIM Manager, ou, a incorporação das funções desse último a função do coordenador de projetos, garantindo mais responsabilidades, e como tal, requer mais conhecimento e técnica, porém representa um grande avanço para o processo de projeto e para o produto final.

\section{METODOLOGIA}

A metodologia utilizada para esta pesquisa foi o estudo de caso. Esse, foi executado em entrevistas, com um coordenador de projetos, onde foram aplicados questionários, com indagações referentes à implantação do BIM, seus objetivos e interesses e como a implantação afetou o escopo de trabalho do coordenador de projetos: as atividades acumuladas, suprimidas e modificadas, além, é claro, da presença do BIM Manager, e a relação deste profissional com o coordenador de projetos.

A pesquisa foi realizada em uma construtora, de grande porte, responsável por empreendimentos luxuosos na cidade, escolhida não só pela sua trajetória na construção civil, como também por ser uma das poucas que utiliza a plataforma BIM em seus produtos.

Com base na entrevista, foi possível analisar o fluxo de trabalho com o BIM, determinar seus usos e como esse impacta no escopo de trabalho do coordenador de projetos, evidenciando as mudanças ocasionadas pelo uso da plataforma para a empresa e para os profissionais envolvidos.

\section{ESTUDO DE CASO}

\subsection{Empresa}

A empresa escolhida para o estudo possui tem sede em João Pessoa, na Paraíba, e possui 10 anos de experiência no mercado da construção civil, com 19 empreendimentos entregues, todos com referência de excelência e qualidade.

Com projetos assinados por grandes referências nacionais e um grande investimento em produtos de renomada qualidade, a construtora possui reconhecimento e credibilidade dos empreendimentos de alto padrão. 


\subsection{Entrevista}

A empresa possui um coordenador de projetos. Assim, a fim de reunir informações sobre esse profissional e suas funções realizou-se entrevistas, onde foi possível traduzir as informações coletadas através de dois questionários.

No primeiro questionário foram abordados aspectos pessoais e profissionais do coordenador, bem como os dados sobre a inserção do BIM na empresa. Requisitou-se informações de caracterização da empresa, seu ramo de atuação e experiência, posteriormente, informações do profissional, seu cargo, e experiência. Além destes pontos, indagou-se sobre como se deu a implantação BIM, seus usos e motivações, e de que maneira se deu a implantação.

O segundo questionário foi caracterizado pelas informações técnicas das funções desempenhadas pelo coordenador de projetos com a inserção do BIM e sobre a presença do BIM Manager na empresa, e como esse se aproxima de sua função. Questionou-se sobre o desempenho do coordenador de projetos com a inserção do BIM, como seu trabalho foi afetado, seu perfil, suas novas demandas e competências para tal função.

\subsection{Resultados e Discussões}

O profissional entrevistado é o único coordenador de projetos da empresa. A ferramenta BIM é utilizada para desenvolvimento, manuseio e compatibilização dos projetos. A inserção da plataforma BIM se deu há 4 meses da realização da pesquisa. Os interesses levados em consideração para tal implantação foram basicamente inovar e reduzir custos.

Na inserção desta nova metodologia de trabalho, a empresa estabeleceu as atividades a serem desenvolvidas com o BIM, a fim de desfrutar de todas as possibilidades que o trabalho em BIM oferece, são elas:

a) Elaboração e concepção de projetos arquitetônico, estrutural e complementares;

b) Elaboração de orçamentos;

c) Elaboração e acompanhamento do cronograma;

d) Design dos sistemas construtivos;

e) Coordenação da construção através do 3D;

f) Controle e planejamento do layout de construção.

Embora definido os usos, identificou-se como grande dificuldade o domínio dos softwares, não só pela falta de treinamento dos funcionários da equipe, como também de alguns projetistas, que não trabalhavam com os softwares que garantem a interoperabilidade do BIM.

Para o coordenador de projetos, a metodologia BIM mostrou-se como um grande auxilio em seu trabalho, trazendo melhorias, proporcionando mais celeridade ao processo de trabalho, como descrito na entrevista, por exemplo, a compatibilização de projetos tornou-se muito mais rápida e ágil. Além de tal constatação, o profissional também deve possuir novos conhecimentos para desempenhar com excelência seu papel com essa nova tecnologia, tais como o domínio dos programas utilizados e um bom conhecimento do processo construtivo, devido as demandas dos softwares utilizados. 
O BIM Manager, figura significativa na implantação BIM, não se faz presente na empresa, passando suas atividades para a função do coordenador de projetos. Entre as atribuições mais consideráveis para a função, tem-se:

a) Estabelecer e acordar um plano de execução BIM, controlando os direitos de acesso dos usuários;

b) Facilitar a coordenação do modelo, promovendo reuniões e análises de interferências;

c) Receber e coordenar a troca de novos modelos, e validar os arquivos;

d) Tomar as preocupações necessárias para garantir que não ocorram problemas de interoperabilidade;

e) Determinar as convenções a serem seguidas para o processo de revisão dos modelos BIM;

f) Estabelecer um protocolo de segurança de dados, fazer backups regulares e estabelecer processamento de rotinas para garantir a segurança do modelo de dados.

Ademais das atividades estabelecidas para o seu uso, o BIM permite gerar e controlar os indicadores de projetos, marca registrada da empresa para avaliação da qualidade de seus projetos. O coordenador de projetos ainda repassa as orientações para a modelagem a ser desenvolvida, a fim de que ela seja compatível com as exigências da construtora.

Cabe destacar também os pontos positivos identificados pela adoção do BIM ao processo de trabalho do coordenador de projetos:

a) Aumento da produtividade dos projetistas;

b) Ganho de tempo na análise e compatibilização de projetos;

C) Maior qualidade dos projetos;

d) Ganho de tempo em orçamentos.

Por fim, não se detectou alterações na metodologia de trabalho do coordenador de projetos, apenas melhorias e atividades incorporadas ao seu processo de trabalho.

\section{CONSIDERAÇÕES FINAIS}

Com a pesquisa, pode-se verificar que, apesar da metodologia BIM ser inovadora e positiva em todo o processo do projeto (e execução), seu uso ainda não está muito difundido no mercado.

Em relação aos impactos desta metodologia, verificou-se mais eficiência e agilidade na compatibilização dos projetos, bem como na avaliação dos seus indicadores. Em contraponto, também pode-se notar a falta de conhecimento com a tecnologia de alguns profissionais, fato este que pode se tornar um limitador no mercado.

No tocante ao desempenho do coordenador de projetos frente a esta metodologia, foi verificado um auxílio em seu processo de trabalho, proporcionando mais produtividade em suas atividades. 
Finalmente, espera-se, com esta pesquisa e sua divulgação, estimular ainda mais o advento do BIM, e que as pesquisas acadêmicas sobre esta temática se perpetuem para embasar ainda mais o uso e difusão desta metodologia na indústria da arquitetura, engenharia e construção.

\section{REFERÊNCIAS}

BARISON, M. B.; SANTOS, E. T. Na overviwm of BIM specialists. In: ICCBE International Conference on Computing in Civil and Building Engineering, 2010, Proceedings. 2010. 6P.

CÂMARA BRASILEIRA DA INDÚSTRIA DA CONSTRUÇÃO. Implantação do BIM para construtoras e incorporadoras: Parte 1 - Fundamentos do BIM. Brasília: CBIC, 2016.

FABRÍCIO, M. M. Projeto simultâneo na construção de edifícios. 2002. 329 f. Tese (Doutorado em Engenharia) - Escola Politécnica, Universidade de São Paulo, 2002.

MANZIONE, L. Proposição de uma estrutura conceitual de gestão do processo de projeto colaborativo com o uso do BIM. 2013. $343 \mathrm{f}$. Tese (Doutorado em Engenharia) - Escola Politécnica, Universidade de São Paulo, 2013.

MELHADO, S. B. Gestão, cooperação e integração para um novo modelo voltado à qualidade do processo de projeto na construção de edifícios. 2001 . 254 f. Tese (Livre-docência) - Escola Politécnica, Universidade de São Paulo, 2001.

NÓBREGA JÚNIOR, C. L. Coordenador de projetos de edificações: estudo e proposta para perfil, atividades e autonomia. 2012. $227 \mathrm{f}$. Tese (Doutorado em Engenharia) - Escola Politécnica, Universidade de São Paulo, 2012.

OLEGÁRIO, W. L. C. Revisão das atividades desempenhadas pelo coordenador de projetos frente a tecnologia BIM. 2018. 58f. Trabalho de Conclusão de Curso (Graduação em Engenharia) - Universidade Federal da Paraíba, 2018.

WANDERLEY, A. K. A. S. Premissas para implantação de Building Information Modeling em empresas de projetos e de construção: estudos de caso. 2017. 181 f. Dissertação (Mestrado em Engenharia). Recife: UPE, Escola Politécnica, 2017. 